\title{
Amygdala signals subjective appetitiveness and aversiveness of mixed gambles
}

Gelskov, Sofie V.; Henningsson, Susanne; Madsen, Kristoffer Hougaard; Siebner, Hartwig R.; Ramsoy, Thomas Z.

\section{Published in:}

Cerebral Cortex

Link to article, DOI:

10.1016/j.cortex.2015.02.016

Publication date:

2015

Document Version

Publisher's PDF, also known as Version of record

Link back to DTU Orbit

Citation (APA):

Gelskov, S. V., Henningsson, S., Madsen, K. H., Siebner, H. R., \& Ramsoy, T. Z. (2015). Amygdala signals subjective appetitiveness and aversiveness of mixed gambles. Cerebral Cortex, 66, 81-90.

https://doi.org/10.1016/j.cortex.2015.02.016

\section{General rights}

Copyright and moral rights for the publications made accessible in the public portal are retained by the authors and/or other copyright owners and it is a condition of accessing publications that users recognise and abide by the legal requirements associated with these rights.

- Users may download and print one copy of any publication from the public portal for the purpose of private study or research.

- You may not further distribute the material or use it for any profit-making activity or commercial gain

- You may freely distribute the URL identifying the publication in the public portal 


\title{
Research report
}

\section{Amygdala signals subjective appetitiveness and aversiveness of mixed gambles}

\author{
Sofie V. Gelskov ${ }^{a, *}$, Susanne Henningsson ${ }^{a, b}$, Kristoffer H. Madsen ${ }^{a, c}$, \\ Hartwig R. Siebner ${ }^{a, d, 1}$ and Thomas Z. Ramsøy ${ }^{a, e, 1}$ \\ ${ }^{a}$ Danish Research Centre for Magnetic Resonance, Centre for Functional and Diagnostic Imaging and Research, \\ Copenhagen University Hospital Hvidoure, Hvidoure, Denmark \\ ${ }^{\mathrm{b}}$ Center for Integrated Molecular Brain Imaging, Copenhagen University Hospital, Denmark \\ c Section for Cognitive Systems, DTU Compute, Technical University of Denmark, Denmark \\ d Department of Neurology, Copenhagen University Hospital Bispebjerg, Copenhagen, Denmark \\ e Center for Decision Neuroscience, Department of Marketing, Copenhagen Business School, Denmark
}

\section{A R T I C L E I N F O}

Article history:

Received 14 October 2014

Reviewed 12 November 2014

Revised 14 January 2015

Accepted 23 February 2015

Action editor Ahmad Hariri

Published online 5 March 2015

Keywords:

Decision-making

Loss aversion

Amygdala

Ventral striatum

fMRI

\begin{abstract}
A B S T R A C T
People are more sensitive to losses than to equivalent gains when making financial decisions. We used functional magnetic resonance imaging (fMRI) to illuminate how the amygdala contributes to loss aversion. The blood oxygen level dependent (BOLD) response of the amygdala was mapped while healthy individuals were responding to 50/50 gambles with varying potential gain and loss amounts. Overall, subjects demanded twice as high potential gain as loss to accept a gamble. The individual level of loss aversion was expressed by the decision boundary, i.e., the gain-loss ratio at which subjects accepted and rejected gambles with equal probability. Amygdala activity increased the more the gainloss ratio deviated from the individual decision boundary showing that the amygdala codes action value. This response pattern was more strongly expressed in loss aversive individuals, linking amygdala activity with individual differences in loss aversion. Together, the results show that the amygdala signals subjective appetitiveness or aversiveness of gain-loss ratios at the time of choice.
\end{abstract}

(C) 2015 Elsevier Ltd. All rights reserved.

\section{Introduction}

When making economic decisions, people often deviate from rational behavior (Kahneman \& Tversky, 1979). For instance, people tend to overestimate the impact of losing, consequently biasing decisions towards loss aversion: when presented with risky gambles with equal chances of winning and losing, people demand on average twice the amount of potential gains compared to losses in order to accept a gamble (Kahneman \& Tversky, 1984; Tom, Fox, Trepel, \& Poldrack, 2007).

Clinical investigations have found diminished loss aversion-bias in amygdala-lesioned patients compared to

\footnotetext{
* Corresponding author. Danish Research Centre for Magnetic Resonance, Section 714, Copenhagen University Hospital Hvidovre, Kettegaard Allé 30, 2650 Hvidovre, Denmark.

E-mail address: gelskov@gmail.com (S.V. Gelskov).

${ }^{1}$ The senior authors contributed equally to the paper. http://dx.doi.org/10.1016/j.cortex.2015.02.016
} 0010-9452/@ 2015 Elsevier Ltd. All rights reserved. 
healthy controls (De Martino, Camerer, \& Adolphs, 2010). How the amygdala influence the willingness to accept gambles is not clear. One possibility is that the amygdala are responding to magnitudes of either gains or losses in order to avoid or deal with aversive events (LeDoux, 2000). In line with this view, a recent loss aversion study reported that amygdala activity reflected magnitudes of single losses, but not single gains (Canessa et al., 2013). However, other neuroimaging studies failed to support an involvement of amygdala in the evaluation of single losses or gains (Sokol-Hessner, Camerer, \& Phelps, 2013; Tom et al., 2007). These studies rather pointed to other dopaminergic meso-cortico-limbic target areas such as the medial orbitofrontal cortex (mOFC) and ventral striatum which process single gain and loss magnitudes.

The classical view that the amygdala are mainly geared to negative events has been recently challenged by neuroimaging studies showing that the amygdala computes both negative and positive stimulus values during value-based decisionmaking (Baxter \& Murray, 2002; Bermudez, Gobel, \& Schultz, 2012; Grabenhorst, Hernadi, \& Schultz, 2012; Jenison, Rangel, Oya, Kawasaki, \& Howard, 2011). The "bivalent" coding of value in the amygdala, which is not specific to negativity or positivity of a stimulus, per se, suggests that the amygdala may track other properties of these value stimuli such as task relevance, the impact or consequence of a choice, or the biological salience of a stimulus. Indeed, several lines of work indicate that the involvement of the amygdala in decisionmaking goes beyond mere value estimation. Functional neuroimaging revealed context dependent activation of the amygdala reflecting whether choices were framed in terms of avoiding losses or seeking gains (De Martino, Kumaran, Seymour, \& Dolan, 2006). Further, amygdala activity was linked to choice-related emotions such as 'relief of a good choice' or 'regret of a bad choice' (Coricelli et al., 2005; Rogan, Leon, Perez, \& Kandel, 2005; Sangha, Chadick, \& Janak, 2013; Seymour et al., 2005). Finally, a series of studies suggest that the amygdala play a key role in evaluating task relevance rather than reflecting the absolute magnitude of value (Bzdok et al., 2011; Ousdal, Reckless, Server, Andreassen, \& Jensen, 2012; Sander, Grafman, \& Zalla, 2003; Wright \& Liu, 2006).

Here, we aimed at resolving the role of amygdala in loss aversive decision-making. We used fMRI to map BOLDresponses in the amygdala of healthy subjects, who decided whether to accept or reject "mixed" (gain-loss) gambles. We manipulated only magnitudes of potential gains and losses, while keeping win-lose probabilities equal (i.e., 50\%). Participants received no feedback on whether they won or lost, creating a decision-context of maximal uncertainty. Interindividual differences in the tendency to weigh losses higher than gains were expressed by the individual decision boundary lambda $(\lambda)$, which represents the gain-loss ratio where subjects on average choose to accept or reject bets with equal probability.

Importantly, in contrast to previous loss aversion studies (Canessa et al., 2013; De Martino et al., 2010; Sokol-Hessner et al., 2013; Tom et al., 2007), each gamble started with a magnitude presentation phase where either the potential loss or gain amount of the gamble was presented alone. This was followed by a decision phase where the full gamble with specified loss and gain amount appeared, and subjects were required to reject or accept the mixed gamble (Fig. 1A). This procedure allowed us to temporally separate neural responses elicited by increasing gain or loss amounts during the first part of the gamble from the assessment of the full mixed gamble defined by the gain-loss ratio during the decision-making phase.

Our study tested two mutually exclusive hypotheses: The amygdala might primarily respond to individual magnitudes of potential monetary losses and/or gains (Belova, Paton, Morrison, \& Salzman, 2007; Belova, Paton, \& Salzman, 2008; Canessa et al., 2013; Paton, Belova, Morrison, \& Salzman, 2006; Salzman, Paton, Belova, \& Morrison, 2007). Alternatively, the amygdala might integrate both gain and lossmagnitudes into the decision process. The latter hypothesis makes the prediction that the amygdala assesses the value of the full gain-loss ratio, relative to the individual decision boundary $\lambda$. If this were the case, amygdala activity might either be tuned to gain-loss ratios far away from the individual decision boundary or preferentially deal with increasingly ambiguous decisions, i.e., gamble ratios that are close to the individual decision boundary.

\section{Materials and methods}

\subsection{Subjects}

Sixteen male subjects (age range 20-32 years; median age 24 years; 9 right handed) participated in the experiment. Two additional subjects were scanned but not analysed, one due to experimenter error, and one due to missing field measurements.

Subjects were screened for any history of neurological or cardiovascular disorders, contra-indications to MRI-scanning and signed health declarations before study commencement. The study was approved under the ethical protocol KF $01-131 / 03$, issued by the local ethics committee. Subjects were added to one of four condition-groups in order to even out unwanted visual or motor artefacts: They either received the gain amount written in orange numerals on the purple half of the pie chart and the loss amount on the orange side with purple numerals, or vice versa. Within this division there were two sub-conditions: a group that answered "yes" with the right index finger, and a group that answered "yes" with right middle finger.

\subsection{Instruction}

Subjects were instructed and trained on the task outside the scanner until they were familiar with it. They were told that after completing the task in the scanner, the computer would randomly draw out three bets, and that all three bets would be played with fifty-fifty chance of winning or losing. They were also reminded that gambling amounts represented real potential losses or gains which after the experiment would be either added to or deducted from their initial participation fee of 350 Danish kroner which corresponds to an endowment of $\approx 58.5$ US Dollars (NB, 1 Danish kroner $\approx .167$ US Dollar. Hereafter $\$$ are used in the text), with a max loss or gain of $\$ 25.1$. In reality, this was a framing setup and all subjects ended up earning between $\$ 20.1-23.4$ in addition to the $\$ 58.5$. 


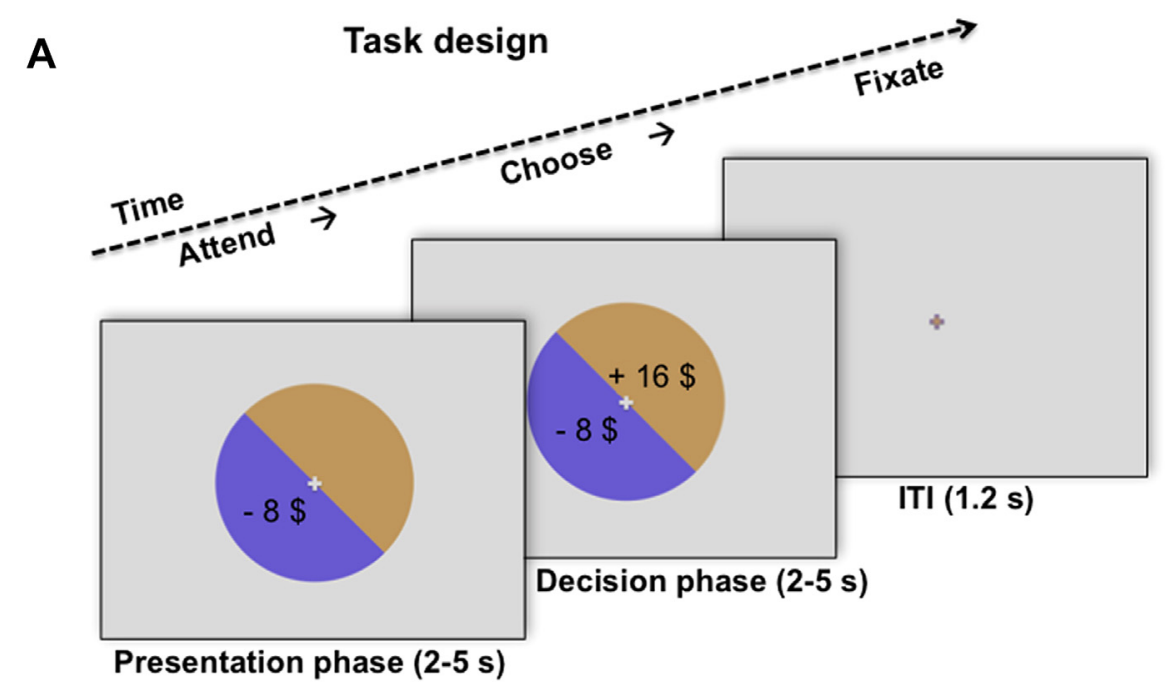

B
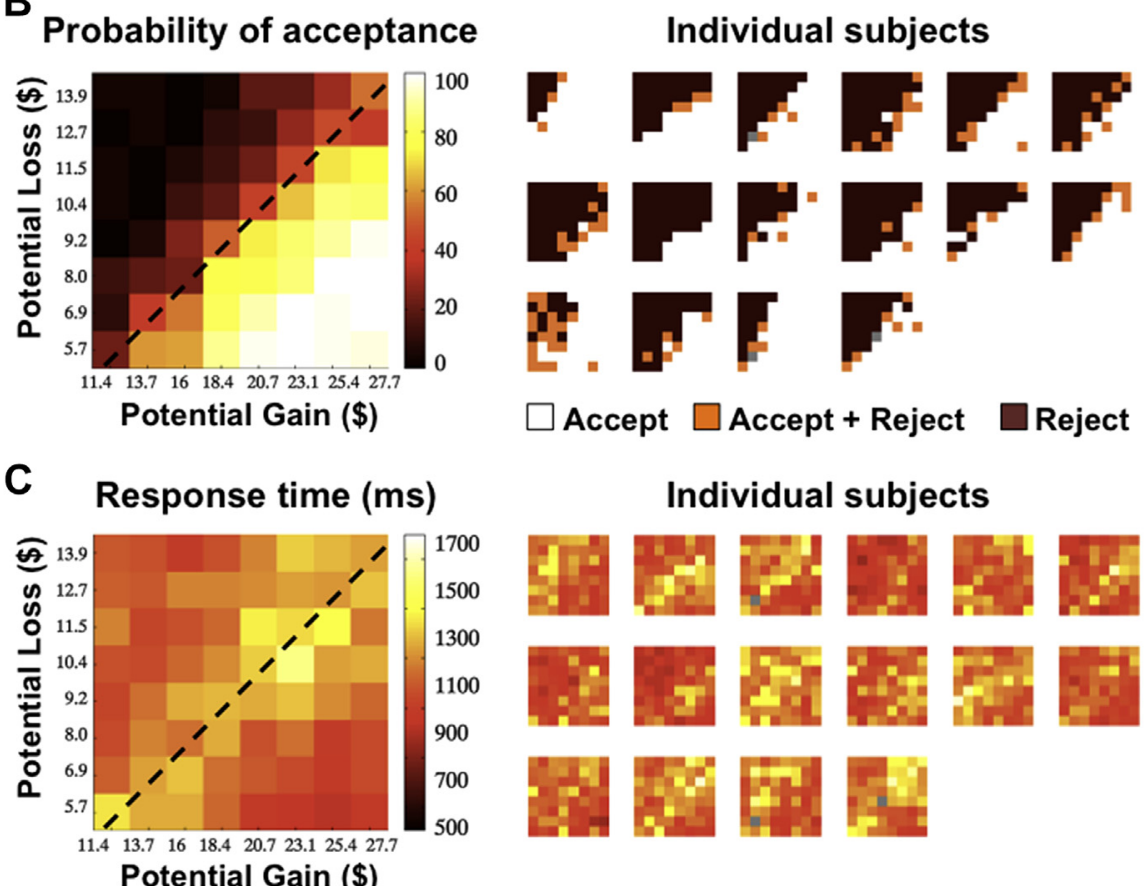

Fig. 1 - Task design and behavioral results. A) Event-related fMRI paradigm; Subjects received potential loss and gain amounts of a given gamble sequentially, with half of the trials starting with potential loss and the other half with potential gain amount. When both potential gain and loss amounts were presented, subjects chose whether to accept or reject the gamble. Inter-trial intervals (ITIs) separated two trials. Stimuli consisted of 64 different gain-loss ratios. All ratios were presented twice in randomized order. Color-coded heat-maps representing: B) choice patterns for group (left) and individuals (right), and C) response time patterns for group and individuals. Color-intensities indicate increasing \% probability of accepting gambles, and increasing response time, respectively. Dotted lines indicate group average decision boundary, $\lambda$, of 2.16. This was the point at which subjects chose to accept and reject gain-loss ratios with equal probability.

\subsection{Stimuli and setting}

Stimuli were projected onto a screen behind the scanner, and consisted of purple and orange pie charts on a uniform gray colour background. Amounts were presented in Danish currency with purple or orange digits. The 64 gain/loss stimuli combined the 8 potential gain amounts (\$11.3-27.8; in increments of $\$ 2.3$ ), with the 8 potential loss amounts (\$5.7-13.9; in increments of \$1.7). Each of the stimuli belonged to one of 8 classes, identified by the angle of the pie chart which was rotated with $45^{\circ}\left(0^{\circ}-360^{\circ}\right)$ for each class. Thus, although each amount (e.g., $+\$ 16$ ) appeared 16 times, it was 
only presented once in the same physical position on the screen per main condition (gain or loss first), as to avoid any visual repetition-effects.

The 64 mixed gambles were presented once in a 'gain first' and once in a 'loss first' condition, yielding a total of 128 trials. To ensure that subjects were attentive to the task and to increase the amount of ratios below 1, we added 18 highly disadvantageous 'catch' trials. These trials combined 3 low gain amounts (i.e., $\$ 5.7,6.9,8$ ) with 3 high loss amounts (i.e., $\$ 23.1,25.4,27.8)$. All subjects rejected at least $89 \%$ of the catch trials, indicating that subjects payed attention to the task. Finally, we added 24 'base-line' trials: "empty" purple/orange pie charts with no letters or numbers with the exception of a central fixation cross. When these randomly distributed trials appeared, subjects had been instructed to wait passively until the next trial. Stimuli were presented and button presses recorded using the E-Prime 2.0 software (Psychology Software Tools, Pittsburgh, PA).

\subsection{Study procedure}

On each trial, subjects were presented with a pie chart with either a potential gain amount or a potential loss amount, according to main condition. After a varying display time $(2-5 \mathrm{sec})$, the second amount of the mixed gamble was presented (see Fig. 1A) and subjects used right index and middle fingers to either accept or reject the bet by pressing a button.

Subjects were instructed to answer as fast as possible, and that there was no correct or incorrect strategy for choosing: They should simply follow their 'gut-feeling' when choosing. The 170 trials were randomly distributed over two runs each lasting $11 \mathrm{~m} 36 \mathrm{sec}$, with an average trial length of $8.2 \mathrm{sec}$. Between each run we performed a short $B_{0}$ mapping scan, allowing the participants a few minutes of rest.

\subsection{Imaging methods}

Functional and structural MR scans were collected using a Siemens Magnetom Trio 3T MR scanner with an 8-channel head coil (Invivo, FL, USA) at Copenhagen University Hospital Hvidovre, Copenhagen, Denmark. Subjects' heads were stabilized by foam padding to reduce motion artefacts. We collected 295 functional volumes using a whole brain $\mathrm{T}^{*}$ weighted echo-planar imaging (EPI) sequence (41 slices; repetition time (TR): $2430 \mathrm{msec}$; echo time (TE): $30 \mathrm{msec}$; flip angle: $90^{\circ}$; field of view (FOV): $192 \mathrm{~mm}$, horizontal plane). The first two volumes were discarded as dummy scans to allow the field to reach steady state. High-resolution 3D full brain magnetization prepared rapid acquisition gradient echo (MPRAGE) structural scans were acquired for the purpose of spatial normalization and co-registration as well as for creating a visualisation template to be used in the figures (1 $\mathrm{mm}$ isotropic voxels; FOV: $256 \mathrm{~mm}$; acquisition matrix $256 \times 256$; TR: 1540; TE: $3.93 \mathrm{msec}$, inversion time: $800 \mathrm{msec}$, flip-angle: $\left.9^{\circ}\right)$. We included $B_{0}$ field measurement after each EPI recording (TR: $488 \mathrm{msec}$; TE: $6.16 \mathrm{msec})$, with 33 slices oriented as defined in the EPI scan $(3 \times 3 \times 3 \mathrm{~mm}$ slices, no interslice gap, 20 oblique to transverse plane).

\subsection{Behavioral analysis}

We calculated the subject specific lambda $(\lambda)$ measure, by fitting a logistic regression model to each participant's binary response (accept/reject) with gain-loss ratio as the independent variable. In this model $\lambda$ indicated the gain-loss ratio for which the probability of accepting a trial was equal to the probability of not accepting a trial.

\subsection{Imaging analysis}

All analysis was carried out using SPM8 (Wellcome Department of Cognitive Neurology, UCL, http://www.fil.ion.ucl.ac. $\mathrm{uk} / \mathrm{spm})$. The pre-processing of imaging data included spatial realignment to the mean image, normalization to an MNI template image, smoothing using an isotropic $8 \mathrm{~mm}$ FWHM Gaussian kernel, and high-pass temporal filtering (cutoff frequency $1 / 128 \mathrm{~Hz}$ ). A Volterra expansion of the estimated head motion parameters, as well as aliased Fourier expansions of the cardiac- and respiration cycles were included as regressors of no interest in the first level general linear model (GLM) (Lund, Madsen, Sidaros, Luo, \& Nichols, 2006). In addition, we included regressors for catch trials, error trials (i.e., $250 \mathrm{msec}>\mathrm{RT}>2500 \mathrm{msec}$ and trials with no answer) and regressors modeling out the motor activation related to finger button presses.

For all regions outside the amygdala, BOLD signal changes were considered statistically significant when they exceeded a family-wise error (FWE) whole brain corrected cluster threshold of $p \leq .05$, using an entry threshold of $p \leq .001$. Trends were reported only if below a threshold of $p \leq .001$, without correction for multiple comparisons. Due to our a priori hypothesis regarding the involvement of the amygdala in loss aversion, we performed small volume corrections (SVC) on BOLD data using an anatomical ROI mask of the bilateral amygdala from Wfu PickAtlas (Maldjian, Laurienti, Kraft, \& Burdette, 2003). For brain regions where we used SVC (i.e., the amygdala), we applied the FWE method at the voxel level to correct for multiple comparisons. Other areas of interest believed to play a role in loss aversion and decision-making are reported at trend level: 1. Striatum, 2. mOFC/vmPFC, 3. Midbrain, 4. Anterior insula, 5. Anterior Cingulate Cortex (ACC; Tom et al., 2007; Canessa et al., 2013).

\subsubsection{General linear models}

Our main GLM included two time-points of interest: the magnitude presentation phase and the decision phase (Fig. 1A). The magnitude presentation phase was divided into separate "gain events" and "loss events", each modeled with their individual amounts as parametric linear modulations. The decision phase was divided into two main regressors of interest: "appetitive bets" and "aversive bets" relative to the subject specific decision boundary $\lambda$ (i.e., according to whether the trial gain-loss ratio was either larger or smaller than individual $\lambda$, respectively). BOLD signal of each of these two main regressors were then modeled in a linear parametric manner with the absolute difference between the current trial gain/loss ratio and the subject specific decision boundary $\lambda$ (i.e., the euclidian "distance" to $\lambda$ ). The main contrast of 
interest was the absolute distance to $\lambda$ and thus, included joint activity from both of these linear parametric predictors.

We furthermore analysed the decision phase of the main model to reveal activity patterns corresponding to a simple monotonic increase in neural activity with the magnitude of gain-loss ratios (i.e., from very worst to best gain-loss ratio).

In the following section, we describe additional GLMs which were designed to further explore the role of amygdala in loss aversive decision-making. Importantly, the models were post-hoc created to ascertain that the neural signature in amygdala as revealed by the main GLM was not better explained by other properties of the gambles. The new GLMs only differed from the main model in the way they modeled the main predictors of interest in the decision-phase. Presentation phase, catch trials, error regressors etc. were identical throughout the models.

First, to investigate the effect of the order in which gains and losses were presented we constructed a model, where the decision phase was divided according to whether a gain or a loss had been presented first. Second, we constructed a model which included a parametric modulation of the decision phase with 'highest stakes' (i.e., which ever was highest of either the potential gain or two times the potential loss), and a third model which included a modulation with 'joint stakes' (i.e., potential gain + absolute potential loss). Finally, the decision phase of our study was inspired by a previous loss aversion paradigm by Tom et al. (2007), which differentiated activity changes scaled to increasing gain or loss magnitudes during decision-making. In order to emulate the model used by Tom et al. (2007), we included a GLM with three parametric modulations of BOLD activation during the decision phase which separated activation corresponding to: 1) increasing potential gain amount, 2) increasing potential loss amount, and 3) distance of the gain-loss ratio of a given trial to a fixed gain-loss ratio (i.e., a $\lambda$ of 2).

\section{Results}

\subsection{Behavioral results}

All subjects weighted potential losses higher than potential gains with a $\lambda$-value above 1 (mean $\lambda=2.16$; median $\lambda=2.08$; range of individual $\lambda$-values: 1.38-2.92). This is consistent with previous studies, which reported mean $\lambda$-values around 2 (Tom et al., 2007; Tversky \& Kahneman, 1992). Decision time increased with ambiguity of the bet. The more the gain-loss ratio of a gamble approached the individual decision boundary $\lambda$, the more time subjects needed to decide whether or not to accept the gamble $\left(p<.001, R^{2}=.12\right.$; see also Fig. $\left.1 C\right)$.

\subsection{Imaging results}

3.2.1. The role of the amygdala in weighting mixed gambles Given our region-specific a priori hypothesis, we primarily examined task-related activity as reflected by the BOLD response in the amygdala. We first addressed the hypothesis that amygdala integrates gains and losses to estimate the full gamble ratio during decision-making. We evidenced changes in neural activity reflecting the distance between a given trial gain-loss ratio and the individual decision boundary $\lambda$ (Left: $\mathrm{x}=-24, \mathrm{y}=-6, \mathrm{z}=-18, P_{\mathrm{SVC}}<.001, \mathrm{Z}=4.96$; Right: $\mathrm{x}=32$, $\mathrm{y}=4, \mathrm{z}=-26, \mathrm{P}_{\mathrm{SVC}}=.017, \mathrm{Z}=3.73$; see Fig. 2 and Table 1 ).

An additional exploratory whole-brain analysis identified several significant clusters where decision-related activity increased in proportion to the distance between the gain-loss ratio of the mixed gamble and the subjective decision boundary $\lambda$. These clusters were located in bilateral mid- and superior temporal gyri, mid- and posterior parts of insula, and pre-, para- and post-central gyri (Table 1). Conversely, clusters in the midbrain $\left(\mathrm{x}=8, \mathrm{y}=-12, \mathrm{z}=-4, p_{\text {uncorr }}<.001, \mathrm{z}=4.09\right)$, left ventral caudate nucleus $\left(\mathrm{x}=-10, \mathrm{y}=14, \mathrm{z}=-4, p_{\text {uncorr }}<.001\right.$, $\mathrm{Z}=3.50)$, right anterior insula $\left(\mathrm{x}=28, \mathrm{y}=20, \mathrm{z}=-4, p_{\text {un- }}\right.$ corr <.001, $Z=3.54)$ as well as in right ACC $(x=6, y=24, z=42$, puncorr $<.001$, $\mathrm{Z}=3.50$ ) showed opposite trends towards a gradual increase in neural activity the more the gain-loss ratio approached the individual decision boundary.

We also tested whether the response profile of the amygdala was driven by either increasingly aversive or appetitive gambles alone. Here, we found that increasingly aversive bets with an unfavorable gamble ratio (relative to the individual $\lambda$ value) yielded a linear increase in BOLD signal in the right amygdala $\left(\mathrm{x}=30, \mathrm{y}=4, \mathrm{z}=-26, p_{\mathrm{svc}}=.004, \mathrm{Z}=4.14\right)$, and a trend in the left amygdala $(x=-26, y=2, z=-16$, $\left.p_{\text {uncorr }}=.001, Z=3.15\right)$. A similar effect was found for increasingly appetitive bets, where the left amygdala displayed a linear increase in activity with increasing distance to the individual $\lambda$-value $\left(\mathrm{x}=-26, \mathrm{y}=-6, \mathrm{z}=-18, p_{\mathrm{SVC}}=.027\right.$, $Z=3.50)$, and a similar trend in the right hemisphere $(x=32$, $\left.\mathrm{y}=-2, \mathrm{z}=-12, p_{\text {uncorr }}=.001, \mathrm{Z}=3.01\right)$. Together, these results show that both increasingly appetitive and aversive bets contribute to the amygdala activation. Although the effect was more pronounced in the left hemisphere for increasingly appetitive gambles and in the right hemisphere for increasingly aversive gambles, the interaction between the two did not yield any changes in activity in the amygdala, even when using a liberal threshold of $p_{\text {uncorr }}<.01$.

The graded increase of amygdala activity with distance from the individual decision boundary $\lambda$ was not influenced by the order in which the gain and loss amount were presented in the preceding magnitude presentation phase, neither regarding changes in BOLD levels nor behaviorally $(F<.001$; $p>$.99).

When confronted with the mixed gambles, amygdala activity was tuned to the distance of the gain-loss ratio from the individual decision boundary $\lambda$. This pattern is compatible with the notion that the amygdala integrates the gain and loss amounts conjointly. However, this response pattern could still be explained by other properties of the bets, such as tracking the "highest stake", i.e., the most salient of the two amounts in the mixed gamble. For instance, if a trial offered a potential monetary loss of $\$ 8$ and a potential gain of $\$ 23.1$, then the amount with highest "impact" would be $\$ 23.1$ (note: in this model, losses were multiplied by 2 to reflect the larger subjective negative value of losses). Another possibility could be that the engagement of the amygdala was tracking "joint stakes" (e.g., if a trial offered a potential loss of $\$ 8$ and a potential gain of $\$ 23.1$, then joint stakes would be: loss + gain $=8+23.1=\$ 31.1$. . To investigate these possibilities we performed two complementary analyses in which we 
A

Left amygdala $[-24,-6,-18]$
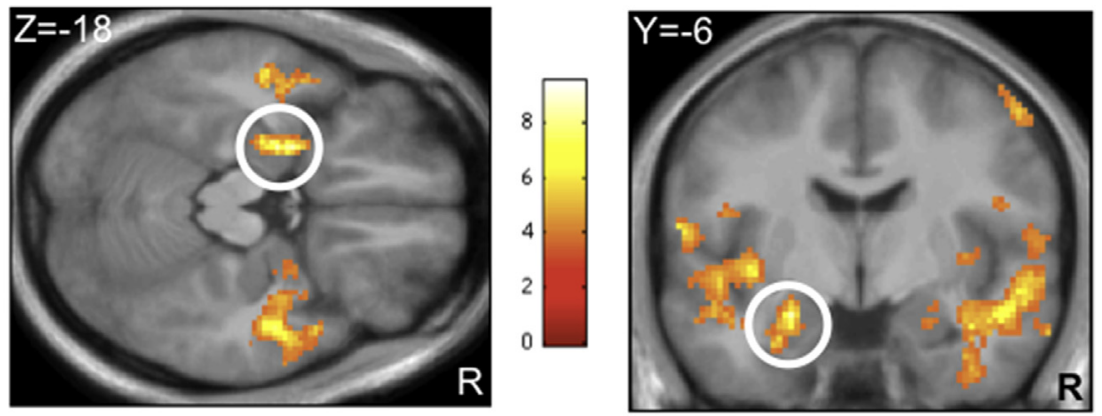

B
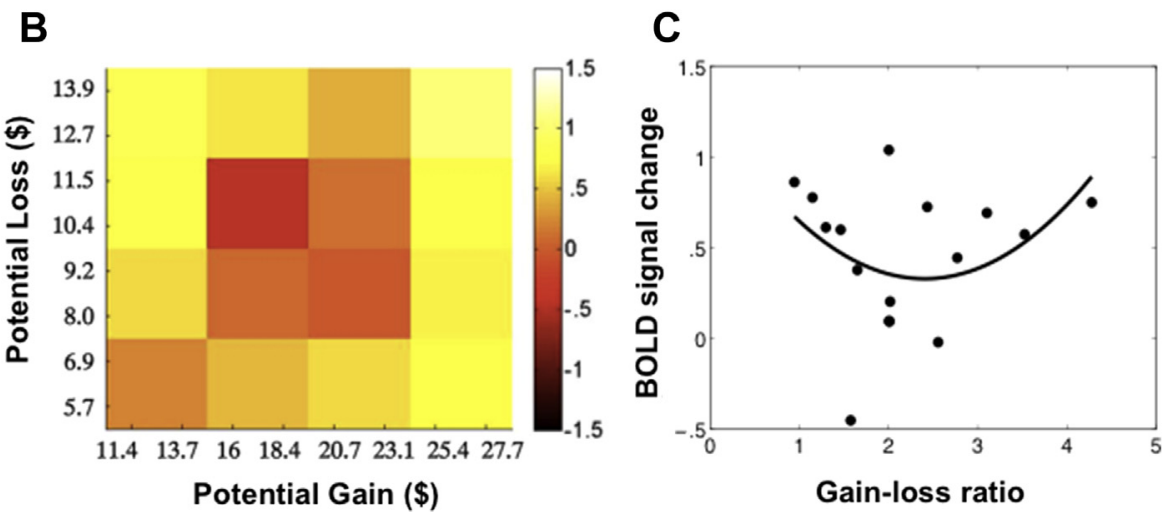

Fig. 2 - Activity profile of the amygdala during the decision-making phase. A) Brain responses reflecting distance to the individual decision boundary $\lambda$. White circle indicates left amygdala cluster $\left(x=-24, y=-6, z=-18, p_{\text {svc }}<.001, z=4.96\right)$. Figure B and C further illustrates the sensitivity of the amygdala to the decision boundary $\lambda$. They are based on a post hoc analysis created for illustrative purposes, which included 16 regressors ("bins") each containing 4 adjacent gain-loss ratios. B) The heat-map illustrates the activation pattern in left amygdala peak (using an 8-voxel sphere), showing that amygdala responses are higher for increasing deviations from the decision boundary. The colors of this heatmap represent strength of activation within each of the 16 bins. C) Response profile of the left amygdala to extreme ratios relative to the intermediate ones around the decision boundary. The $\mathrm{X}$-axis denotes gain to loss ratios in each of the 16 bins of the post hoc analysis. $Y$ values indicate \%-signal change of global mean.

included modeling of the "highest stakes" or the "joint stakes" to our original statistical model. The activity profile in the amygdala was neither reflected by the "highest stake" nor the "joint stakes" of a gamble, even using a liberal threshold of $p_{\text {uncorr }}<.01$.

\subsubsection{Effect of loss aversion}

We were interested in testing whether the individual degree of loss aversion was reflected in the neural activity of the amygdala using the individual $\lambda$ value of participants as a covariate in the second level analysis. Individuals who were more loss averse (i.e., who had a higher $\lambda$ and rejected more

Table 1 - Group results: Clusters showing increases in neural activity (as reflected by the BOLD response) with increasing distance to decision boundary.

\begin{tabular}{|c|c|c|c|c|c|c|}
\hline Cluster size & Anatomical peak region & Hemisphere & $\mathrm{x}$ & $\mathrm{y}$ & $\mathrm{z}$ & Z value \\
\hline \multirow[t]{3}{*}{3317} & Rolandic operculum/insula & $\mathrm{L}$ & -44 & -14 & 20 & 5.22 \\
\hline & Inferior parietal lobule & $\mathrm{L}$ & -62 & -46 & 22 & 4.74 \\
\hline & Middle temporal gyrus & $\mathrm{L}$ & -50 & -12 & -20 & 4.64 \\
\hline \multirow[t]{2}{*}{285} & Amygdala & $\mathrm{L}$ & -24 & -6 & -18 & 4.96 \\
\hline & Hippocampus & $\mathrm{L}$ & -28 & -6 & -26 & 4.06 \\
\hline \multirow[t]{2}{*}{675} & Paracentral lobule & $\mathrm{R}$ & 4 & -30 & 70 & 4.95 \\
\hline & Paracentral lobule & $\mathrm{L}$ & -6 & -38 & 72 & 4.44 \\
\hline 210 & Insula/putamen & $\mathrm{R}$ & 34 & 6 & 8 & 4.91 \\
\hline \multirow[t]{3}{*}{1850} & Middle temporal gyrus & $\mathrm{R}$ & 46 & -10 & -20 & 4.81 \\
\hline & Middle temporal gyrus & $\mathrm{R}$ & 50 & -4 & -16 & 4.51 \\
\hline & Superior temporal gyrus & $\mathrm{R}$ & 50 & 6 & -14 & 4.35 \\
\hline 324 & Angular gyrus & $\mathrm{R}$ & 62 & -60 & 22 & 4.22 \\
\hline
\end{tabular}


gambles) showed a steeper rise in regional activity with increasing distance between the individual decision boundary $\lambda$ and the gain-loss ratio of the gamble. Regions showing this effect were the left ventral striatum $(x=-14, y=8, z=-10$, $p_{\text {cluster }}=.037, Z=4.44$, Fig. $\left.3 \mathrm{~A}\right)$ and the left amygdala $(\mathrm{x}=-22$, $\mathrm{y}=0, \mathrm{z}=-14, p_{\text {SVC }}=.005, \mathrm{Z}=4.08$, Fig. 3B).

We then assessed whether this loss aversion effect was present for both increasingly appetitive and aversive bets. Increasingly appetitive bets were associated with a significant correlation between loss aversion and left amygdala activity $\left(\mathrm{x}=-18, \mathrm{y}=-2, \mathrm{z}=-18, p_{\mathrm{svc}}=.039, \mathrm{z}=3.37\right)$. For increasingly aversive bets, we found trend activation in the right amygdala, $\left(\mathrm{x}=18, \mathrm{y}=4, \mathrm{z}=-18, p_{\text {uncorr }}=.001, \mathrm{z}=3.01\right)$. The difference between the appetitive and aversive bets and their correlation with loss aversion did not yield any significant effects in the amygdala, even at a liberal threshold of $p_{\text {uncorr }}<.01$.

\subsubsection{Neural responses to the magnitude of potential gains} and losses in the decision phase

So far, we have focused on the neural response to the full gain/ loss ratios during the decision-phase. However, we wanted to investigate whether these brain responses could be better explained by the separate magnitude of gains or losses during the decision phase. We emulated the analysis used in Tom et al. (2007), which probed for activity changes in brain regions correlating with either 1 ) increasing gain magnitude, 2) increasing loss magnitude and 3) trial ratio distance to a fixed lambda of 2, during the decision-phase. In accordance with their study, we found a weak trend activation in ventral striatum for increasing potential gain (Supplementary Fig. 1). However, we failed to reproduce the reported deactivation in

A Left ventral striatum $[-14,8,-10]$
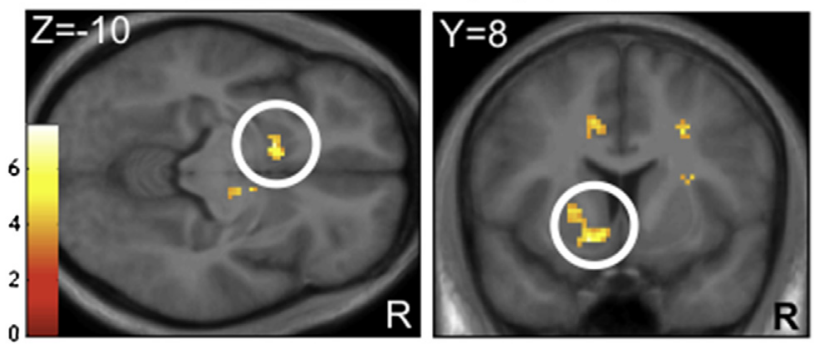

B Left amygdala $[-22,0,-14]$
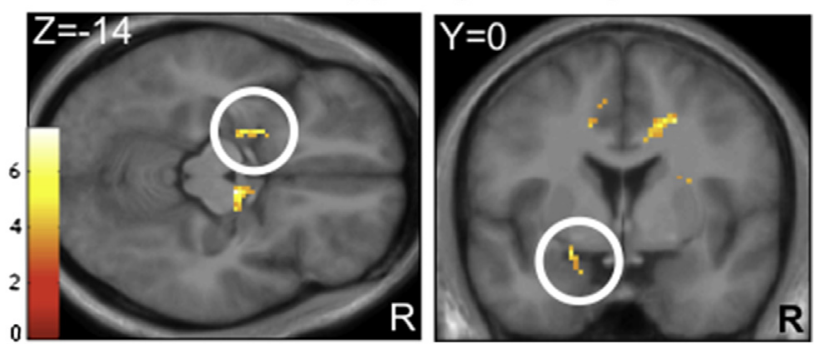

Fig. 3 - Neural correlates of loss aversion during decisionmaking. A) Increased left ventral striatum activation was found for more loss averse individuals to increasingly extreme ratios ( $p_{\text {cluster }}=.037, Z=4.44$ ). B) Stronger responses to higher loss aversion was also found in left amygdala ( $\left.p_{\mathrm{svc}}=.007, \mathrm{z}=4.08\right)$. ventral striatum with increasing potential loss or the mOFC activation and deactivation for gains and losses. Neither magnitude of gains, losses nor increasing distance to a fixed lambda of 2, yielded any amygdala activation during the decision-phase. However, when using the increasing distance to a fixed lambda of 2 as the only parametric modulation of the BOLD response, the left amygdala showed significant activity modulation $\left(\mathrm{x}=-28, \mathrm{y}=2, \mathrm{z}=-22, p_{\mathrm{SVC}}=.033, \mathrm{Z}=3.41\right)$.

\subsubsection{Magnitude of gain-loss ratio}

We performed an additional analysis to identify brain regions where neural activity during the decision phase increased monotonically with the gain-loss ratio of the mixed gambles (from worst to best). A cluster in the dorsal midbrain covering superior colliculus and dorsal raphe region $(x=4, y=-28$, $\mathrm{z}=-8, p_{\text {cluster }}<.001, \mathrm{Z}=4.29$ ) showed gradual increases in activity with increasingly appetitive ratios, as did the caudal portion of the ventral striatum $(\mathrm{x}=2, \mathrm{y}=0, \mathrm{z}=-4$, $p_{\text {cluster }}=.002, Z=4.09$; Fig. 4). We found no reductions in regional BOLD signal with increasing gain-loss ratios, even at a liberal threshold of $P_{\text {uncorr }}<.01$. The individual degree of loss aversion as indexed by $\lambda$ did not influence the activity increase in the midbrain or striatum with increasing appetitiveness of gain-loss ratios. Instead, regional increases in activity with
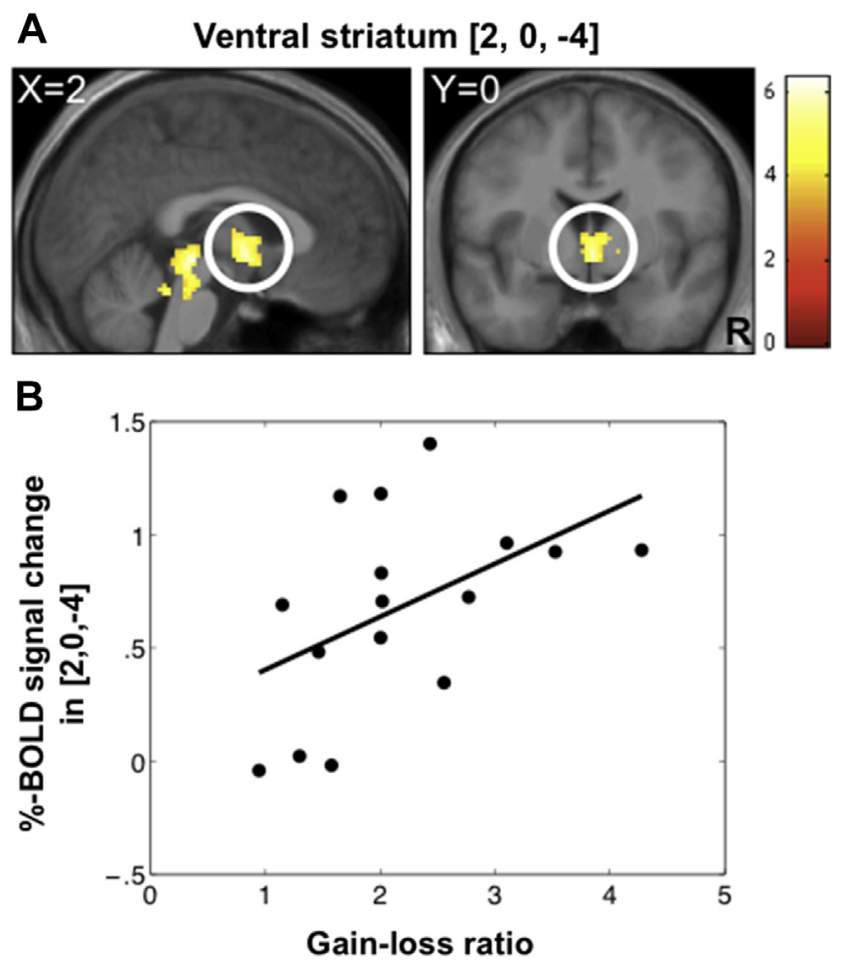

Fig. 4 - Activity profile in ventral striatum during decisionmaking. A) Linear increase in neural activity with the magnitude of gain-loss ratios in a caudal region of the ventral striatum, ( $p_{\text {cluster }}=.002, Z=4.09$ ) and a midbrain cluster ( $p_{\text {cluster }}<.001, z=4.29$ ) covering the superior colliculi and dorsal raphe nucleus. B) Plot of the positive linear relationship between BOLD signal in the ventral striatum (8-voxel sphere) and gamble ratio, based on the post hoc "16-ratio bins" analysis. 
increasing loss aversion were found in the right superior occipital/inferior parietal lobe $\left(\mathrm{x}=30, \mathrm{y}=-70, \mathrm{z}=26, p_{\text {clus }-}\right.$ ter $<.001, Z=4.22)$ and mid-occipital lobe $(x=-22, y=-90, z=-2$, pcluster $=.008, Z=3.79$ ).

\subsubsection{Neural responses to the magnitude of potential gains} and losses in the presentation phase

Passive viewing of potential gains and losses in the presentation phase was investigated by combining the effect of decreasing potential loss and increasing potential gain. This effect of value was reflected in the left anterior insula, as well as a trend in the right anterior insula (Supplementary Fig. 2). Valuation of potential gains and losses also activated the left supra-marginal gyrus and precentral gyrus, right lingual gyrus and precuneus. The left ventral striatum also showed a trend activation $\left(\mathrm{x}=-12, \mathrm{y}=6, \mathrm{z}=-10, p_{\text {uncorr }}<.001, \mathrm{Z}=3.23\right)$. By contrast, there was no activation in the amygdala associated with magnitude estimation during the presentation phase, even when using a lenient threshold of $p_{\text {uncorr }}<.01$. Likewise, the activation in amygdala did not change with magnitude of gains or losses separately. Previous studies have indicated an important role of $\mathrm{mOFC/vmPFC}$ in valuation (e.g., Tom et al., 2007), and by lowering the threshold to $p_{\text {uncorr }}<.01$ we could confirm bilateral trends in the mOFC reflecting increasing gain magnitude (mOFC: $\mathrm{x}=-8, \mathrm{y}=54, \mathrm{z}=-12, p_{\text {uncorr }}<.01$, $Z=3.25)$.

\section{Discussion}

The present study sheds new light on the role of the amygdala in loss averse decision-making. We found that the amygdala assessed the gain-loss ratio of a full bet during a gambling task that required subjects to balance the gain and loss of each bet in relation to subject-specific decision boundaries. In contrast, single gain or loss amounts did not modulate amygdala activity. This implies that the amygdala evaluates a mixed gamble as a whole rather than assessing single magnitudes of individual gains or losses.

We found that amygdala activity during the decision phase was tuned to the individual decision boundary $\lambda$. More precisely, amygdala activity increased the more the gain-loss ratio of a given gamble deviated from the individual decision boundary $\lambda$. The amygdala response to increasingly aversive and increasingly appetitive gambles is in good agreement with previous studies in human and non-human primates that have implicated the amygdala in processing of both appetitive and aversive stimuli during decision-making (Baxter \& Murray, 2002; Breiter, Aharon, Kahneman, Dale, \& Shizgal, 2001; Paton et al., 2006; Yacubian et al., 2006). For instance, single neurons in the amygdala displayed positive and negative linear increases in spiking activity with the values assigned to individual food items, when patients with epilepsy made simple purchase decisions between food items (Jenison et al., 2011). Together, these results indicate that the amygdala plays an important part in integrating subjective appetitiveness and aversiveness of gain-loss ratios at the time of choice.

The individual level of loss aversion (as reflected by the decision boundary $\lambda$ ) had an impact on the amygdala response during decision-making. More loss aversive individuals (with a high $\lambda$ ) put a stronger weight on asymmetric gain-loss ratios that are distant to the individual decision boundary than less loss averse individuals (with a low $\lambda$ ). The relationship between amygdala signaling and the individual expression of loss aversion raises the possibility that the amygdala reinforces a pre-existing bias towards loss aversion. Indeed the amygdala has been implicated in decision biases and guidance of loss averse decisions (Canessa et al., 2013; De Martino et al., 2010; De Martino et al., 2006).

Previous studies show that the amygdala support decisions made under a high degree of uncertainty (Adams, Gordon, Baird, Ambady, \& Kleck, 2003; Herry et al., 2007; Hsu, Bhatt, Adolphs, Tranel, \& Camerer, 2005) and in assessing task relevance (Davis \& Whalen, 2001; Ousdal et al., 2012; Sander et al., 2003; Zhang et al., 2013). In this study, the nature of the gambling task favored an engagement of the amygdala in decision-making: The equal gain-loss probabilities and the absence of feedback introduced maximal uncertainty with respect to gamble outcomes. Although the level of uncertainty was equally high for all bets, amygdala activity was stronger for ratios at both extremes of the valence spectrum. The increasing asymmetry of gain-loss ratios signaled high stakes (i.e., potentially large losses or large gains) and entailed higher potential financial impact. The present results are therefore in agreement with the notion that the amygdala evaluates the impact or relevance of a given choice.

This study is the first indicating a role of the amygdala in balancing the entire gamble, as opposed to only reflecting losses (Canessa et al., 2013), or even playing no role in loss aversion (Tom et al., 2007). In the present study, a framing setup was used to make subjects believe they were risking some of their participation fee, while, in fact, all of the subjects were predestined to keep their fee and even gain extra money by the end of the experiment. However, participants did not know this and they were thus very motivated to keep the monetary participation fee that they had already counted on, something that we believe resulted in a strong "endowment effect" (Kahneman \& Tversky, 1979; Thaler, 1980). In contrast, other loss aversion studies typically endowed participants with money to gamble for (Canessa et al., 2013; Sokol-Hessner et al., 2013; Tom et al., 2007). We believe that our endowment method reflected a more naturalistic setup, resembling real-life financial decisions more closely. This resulted in a consistently high behavioral loss aversion and in increased amygdala engagement when deciding to accept or reject gambles.

Decisions required more effort the closer the gain-loss ratio was to the individual decision boundary $\lambda$, as evidenced by longer deliberation times. This is likely to reflect the higher similarity of subjective utilities for gains and losses, resulting in more difficult decisions around the decision boundary. The activity profile expressed by the amygdala is incompatible with the claim that this region is tracking increasing choice ambiguity. The results rather show the opposite, namely an engagement of the amygdala in intuitively clear decisions, facilitating fast decisions towards appetitive situations and away from aversive ones. Difficulty of decision-making in stead engaged areas, which are believed to monitor conflict 
such as the anterior insula and ACC (FitzGerald, Seymour, \& Dolan, 2009).

Although we have mainly focused on the amygdala, it should be noted that decisions were also influenced by a larger network of regions including somatosensory components (rolandic operculum, posterior insula and paracentral lobule) and limbic components (hippocampus, amygdala and putamen). A similar functional network was recently hypothesized to mediate an aversive signal that could bias decision-making towards avoiding losses during mixed gambles (Canessa et al., 2013).

Here, we found that additional regions (i.e., inferior parietal lobule, superior temporal gyrus and angular gyrus) also influenced decisions. These latter regions have been linked to reading and numerical computations (e.g., Carreiras et al., 2009; Dehaene, Spelke, Pinel, Stanescu, \& Tsivkin, 1999; Hickok \& Poeppel, 2007) and could have the important function of deciphering amounts (e.g., “-\$8” us “+\$16”) and calculating approximate gamble ratios based on these amounts.

Our mixed-gamble task included a magnitude presentation phase and a decision-making phase. This allowed us to investigate whether the amygdala separately or jointly processes the gain and loss amounts of mixed gambles. The amygdala did not respond to changes in stimulus value in the passive presentation phase, neither when investigating the whole spectrum from worst to best amount, nor for changes in either gain or loss-magnitudes separately. However, regional trends in the ventral striatum and the mOFC reflected increasing value and gain magnitude, respectively. Activity in these specific regions indicate that neural computations in this phase likely reflect valuation (e.g., Tom et al., 2007).

In the active decision-making phase, amygdala activity did not code the gain or loss magnitude that determined the absolute gain-loss ratio of the gamble. The amygdala also did not reflect the absolute gain-loss ratio irrespective of subject-specific $\lambda$ values. Instead, we found the amygdala to be specifically involved in balancing gain-loss ratios relative to the subjective decision boundary. As distance increased between gain-loss ratio and the individual decision boundary, amygdala activity became stronger. Under those conditions, the involvement of the amygdala correlated nicely with the degree of individual loss-aversion biases, extending previous studies that have implicated the amygdala in loss processing and behavioral loss aversion (Canessa et al., 2013; De Martino et al., 2010; Sokol-Hessner et al., 2013; Yacubian et al., 2006). Since amygdala activation was absent during the magnitude presentation phase, it is unlikely that amygdala activity in the decision phase was confounded by "spill-over" amygdala activity from the prospect phase.

Together with the amygdala, the ventral striatum was tuned to the gradual increase in subjectively aversive and appetitive gambles in more loss averse subjects. We speculate that loss averse subjects may have an enhanced reliance on choice-related emotions (so-called "gut-feelings") which would help them avoid unfavorable situations (i.e., losing large amounts of money) and approach favorable situations (i.e., gaining large amounts) (Coricelli et al., 2005; Rogan et al., 2005; Sangha et al., 2013; Seymour et al., 2005). In the absence of actual feedback, this could assist individuals to navigate in their personalized 'decision-space' of gain-loss ratios. However, further research is needed to explain the origin of this stronger reliance on the amygdala in more biased subjects.

In conclusion, our results significantly expand previous work by showing a consistent role of the amygdala in delineating the subjective decision-space. Importantly, the present results point to a primary engagement of the amygdala in actual decision-making, since it was active only after subjects obtained complete information about the gain-loss ratio.

\section{Acknowledgments}

This work was supported by the The Danish Council for Independent Research in Social Sciences through a grant to Thomas Ramsøy (no. 0601-01361B) and by the Lundbeck Foundation through a Grant of Exellence (no. R59 A5399) to Hartwig Siebner. We thank all the participants for their time. We thank Sid Kouider for helpful comments on the manuscript, Martin Skov for initial discussions, Helle Ruff Laursen for assistance during scanning and two anonymous reviewers for their helpful comments on the manuscript.

Hartwig Siebner has received honoraria as speaker from Lundbeck A/S, Valby, Denmark, Biogen Idec, Denmark A/S, Genzyme, Denmark and MerckSerono, Denmark, honoraria as editor from Elsevier Publishers, Amsterdam, The Netherlands and Springer Publishing, Stuttgart, Germany, travel support from MagVenture, Denmark, and grant support from Biogen Idec, Denmark A/S. The other authors declare no competing financial interests.

\section{Supplementary data}

Supplementary data related to this article can be found at http://dx.doi.org/10.1016/j.cortex.2015.02.016.

\section{R E F E R E N C E S}

Adams, R. B., Jr., Gordon, H. L., Baird, A. A., Ambady, N., \& Kleck, R. E. (2003). Effects of gaze on amygdala sensitivity to anger and fear faces. Science, 300, 1536.

Baxter, M. G., \& Murray, E. A. (2002). The amygdala and reward. Nature Reviews Neuroscience, 3, 563-573.

Belova, M. A., Paton, J. J., Morrison, S. E., \& Salzman, C. D. (2007). Expectation modulates neural responses to pleasant and aversive stimuli in primate amygdala. Neuron, 55, 970-984.

Belova, M. A., Paton, J. J., \& Salzman, C. D. (2008). Moment-tomoment tracking of state value in the amygdala. Journal of Neuroscience, 28, 10023-10030.

Bermudez, M. A., Gobel, C., \& Schultz, W. (2012). Sensitivity to temporal reward structure in amygdala neurons. Current Biology, 22, 1839-1844.

Breiter, H. C., Aharon, I., Kahneman, D., Dale, A., \& Shizgal, P. (2001). Functional imaging of neural responses to expectancy and experience of monetary gains and losses. Neuron, 30, 619-639.

Bzdok, D., Langner, R., Caspers, S., Kurth, F., Habel, U., Zilles, K., et al. (2011). ALE meta-analysis on facial judgments of 
trustworthiness and attractiveness. Brain Structure \& Function, 215, 209-223.

Canessa, N., Crespi, C., Motterlini, M., Baud-Bovy, G., Chierchia, G., Pantaleo, G., et al. (2013). The functional and structural neural basis of individual differences in loss aversion. Journal of Neuroscience, 33, 14307-14317.

Carreiras, M., Seghier, M. L., Baquero, S., Estévez, A., Lozano, A., Devlin, J. T., et al. (2009). An anatomical signature for literacy. Nature, 461, 983-986.

Coricelli, G., Critchley, H. D., Joffily, M., O'Doherty, J. P., Sirigu, A., \& Dolan, R. J. (2005). Regret and its avoidance: a neuroimaging study of choice behavior. Nature Neuroscience, 8, 1255-1262.

Davis, M., \& Whalen, P. J. (2001). The amygdala: vigilance and emotion. Molecular Psychiatry, 6, 13-34.

De Martino, B., Camerer, C. F., \& Adolphs, R. (2010). Amygdala damage eliminates monetary loss aversion. Proceedings of the National Academy of Sciences of the United States of America, 107, 3788-3792.

De Martino, B., Kumaran, D., Seymour, B., \& Dolan, R. J. (2006). Frames, biases, and rational decision-making in the human brain. Science, 313, 684-687.

Dehaene, S., Spelke, E., Pinel, P., Stanescu, R., \& Tsivkin, S. (1999). Sources of mathematical thinking: behavioural and brainimaging evidence. Science, 284, 970-974.

FitzGerald, T. H., Seymour, B., \& Dolan, R. J. (2009). The role of human orbitofrontal cortex in value comparison for incommensurable objects. Journal of Neuroscience, 29, 8388-8395.

Grabenhorst, F., Hernadi, I., \& Schultz, W. (2012). Prediction of economic choice by primate amygdala neurons. Proceedings of the National Academy of Sciences of the United States of America, 109, 18950-18955.

Herry, C., Bach, D. R., Esposito, F., Di Salle, F., Perrig, W. J., Scheffler, K., et al. (2007). Processing of temporal unpredictability in human and animal amygdala. Journal of Neuroscience, 27, 5958-5966.

Hickok, G., \& Poeppel, D. (2007). The cortical organization of speech processing. Nature Reviews Neuroscience, 8, 393-402.

Hsu, M., Bhatt, M., Adolphs, R., Tranel, D., \& Camerer, C. F. (2005). Neural systems responding to degrees of uncertainty in human decision-making. Science, 310, 1680-1683.

Jenison, R. L., Rangel, A., Oya, H., Kawasaki, H., \& Howard, M. A. (2011). Value encoding in single neurons in the human amygdala during decision making. Journal of Neuroscience, 31 , 331-338.

Kahneman, D., \& Tversky, A. (1979). Prospect theory - analysis of decision under risk. Econometrica, 47, 263-291.

Kahneman, D., \& Tversky, A. (1984). Choices, values, and frames. American Psychologist, 39, 341-350.

LeDoux, J. E. (2000). Emotion circuits in the brain. Annual Review of Neuroscience, 23, 155-184.
Lund, T. E., Madsen, K. H., Sidaros, K., Luo, W. L., \& Nichols, T. E. (2006). Non-white noise in fMRI: does modelling have an impact? NeuroImage, 29, 54-66.

Maldjian, J. A., Laurienti, P. J., Kraft, R. A., \& Burdette, J. H. (2003). An automated method for neuroanatomic and cytoarchitectonic atlas-based interrogation of fMRI data sets. NeuroImage, 19, 1233-1239.

Ousdal, O. T., Reckless, G. E., Server, A., Andreassen, O. A., \& Jensen, J. (2012). Effect of relevance on amygdala activation and association with the ventral striatum. NeuroImage, 62, 95-101.

Paton, J. J., Belova, M. A., Morrison, S. E., \& Salzman, C. D. (2006). The primate amygdala represents the positive and negative value of visual stimuli during learning. Nature, 439, 865-870.

Rogan, M. T., Leon, K. S., Perez, D. L., \& Kandel, E. R. (2005). Distinct neural signatures for safety and danger in the amygdala and striatum of the mouse. Neuron, 46, 309-320.

Salzman, C. D., Paton, J. J., Belova, M. A., \& Morrison, S. E. (2007). Flexible neural representations of value in the primate brain. Annals of the New York Academy of Science, 1121, 336-354.

Sander, D., Grafman, J., \& Zalla, T. (2003). The human amygdala: an evolved system for relevance detection. Reviews Neuroscience, 14, 303-316.

Sangha, S., Chadick, J. Z., \& Janak, P. H. (2013). Safety encoding in the basal amygdala. Journal of Neuroscience, 33, 3744-3751.

Seymour, B., O'Doherty, J. P., Koltzenburg, M., Wiech, K., Frackowiak, R., Friston, K., et al. (2005). Opponent appetitiveaversive neural processes underlie predictive learning of pain relief. Nature Neuroscience, 8, 1234-1240.

Sokol-Hessner, P., Camerer, C. F., \& Phelps, E. A. (2013). Emotion regulation reduces loss aversion and decreases amygdala responses to losses. Social Cognitive and Affective Neuroscience, 8, 341-350.

Thaler, R. (1980). Toward a positive theory of consumer choice. Journal of Economic Behavior \& Organization, 1, 39-60.

Tom, S. M., Fox, C. R., Trepel, C., \& Poldrack, R. A. (2007). The neural basis of loss aversion in decision-making under risk. Science, 315, 515-518.

Tversky, A., \& Kahneman, D. (1992). Advances in prospect-theory - cumulative representation of uncertainty. Journal of Risk and Uncertainty, 5, 297-323.

Wright, P., \& Liu, Y. (2006). Neutral faces activate the amygdala during identity matching. NeuroImage, 29, 628-636.

Yacubian, J., Glascher, J., Schroeder, K., Sommer, T., Braus, D. F., \& Buchel, C. (2006). Dissociable systems for gain- and lossrelated value predictions and errors of prediction in the human brain. Journal of Neuroscience, 26, 9530-9537.

Zhang, W., Schneider, D. M., Belova, M. A., Morrison, S. E., Paton, J. J., \& Salzman, C. D. (2013). Functional circuits and anatomical distribution of response properties in the primate amygdala. Journal of Neuroscience, 33, 722-733. 